\title{
Về Một Bài Toán Thiết Kế Phương Án Minimax Kiềm Tra Nghiệm Thu Thống Kê
}

\author{
Nguyễn Thanh Tùng \\ Viện Tin Học \\ Viện Khoa Học Việt Nam
}

\section{Mớ đầu}

Khi thiết kế plương án kiềm tra nghiệm thu thơng kê, nếu có đảy đủ cà hai loại thông tin: thóng tin về các tôn thát và thông tin về sự biến động của chất lượng trung bỉnh của sả" xuất thì phırơng pháp Bayes là phırơng pháp thích hợp nhất. Trường hợp không có thông tin vè sự biến động của chát lượng trung bỉnh, người ta bằng lòng với phương pháp thiết kể dựa trên nguyên lý minimax.

Trong những năm qua, bài toán thiét kế phương án kiểm tra nghiệm thu thống kê dựa trên nguyên lý minimax (phương án minimax) được nhièu người quan tâm tới, (xem chẳng hạn [1], [3], [7]). Tuy vậy các cóng trình nghiên cứu cho tới nay chỉ giới hạn trong khuân khổ mô hình bài toán với nhưng giả thićt tỉnh huống khá đơn giản như sau:

- Quá trình sản xuát được dặc trưng bằng một quá trỉnh ngẫu nghiên dùng.

- Các tổn thát trên mọt đơn vị sản phẩm ứng với các quyết định clấp nhận hay bảc bỏ là những hàm tuyến tính của chát lượng lò.

- Phí tồn lấy mẫu kiĉ̉m tra chỉ tỉ lệ với sổ dơn vị sản phẩm mẫu mà không phụ thuộc vào tỉnh trạng chất lượng lô.

Bài này nhằn trình bày mô hỉnh một bài toán thiết kế phương án minimax kiểm tra nghiệm thu thóng kê với những giả thiết tỉnh huóng khá mểm dẻo; chúng minh một số tính chất của plương án minimax, trên cơ sở dó dưa ra thuật toán hữu hiệu xác định nó. 


\section{Mô hình bài toán và nguyên lý minimax xác định phương án kiểm tra}

Giả sử cản xây dựng phương án lấy mẫu một lẩn $(n, c)$ kiểm tra một loạt ô sản phẩm có cỡ $N$ nào đó trong tình huống giả thiết sau đây:

a) Mỗi đơn vị sản phầm chỉ có thể là chính sản phâm hoặc thứ phẩm. Quá trình sản xuất là một quá trỉnh chịu sự điều chỉnh Bernoulli với tham số $p$ (chất lượng trung bỉnh của sản xuất) không đổi trong phạm vi mỗi lô nhưng thay đổi tị̛ ló này sang lô khảc với phân bố xác suất không biết.

b) Dựa trên kết quả kiềm tra mẫu, hai quyết định có thể chọn là: chấp nhận hay bác bỏ phân còn lại không kiêm tra của lô.

c) Không những tổn thát do chấp nhận hay bác bỏ một đơn vị sả phẩm mà cả phí tồn kiểm tra mẫu cũng là những hàm tuyến tính của tỉ lệ thứ phẩm $\rho$ trong lô và đượ lần lượt ký hiệu bới:

$$
\begin{gathered}
k_{a}(\rho)=A_{1}+A_{2} \rho, \\
k_{r}(\rho)=R_{1}+R_{2} \rho, \\
k_{s}(\rho)=s_{1}+s_{2} \rho .
\end{gathered}
$$

Rõ ràng, khi kiểm tra là có sửa chữa thì giả thiết này sẽ hợp lý hơn giả thiết phí tồn kiềm tra chì tỉ lệ với cỡ mẫu.

Có thể xem các hệ só $A_{1}, R_{1}$, và $S_{1}$ làn lượt là tổn thất của việc chấp nhận, bác bỏ và kiêm tra một đơn vị sán phẩm khi đơn vị sản phẩm đó là chính phẩm, còn $A_{2}, R_{2}$, và $S_{2}$ làq tổn thất cũng của các sự việc đó nhưng trong trường hợp đơn vị sản phẩm là thứ phẩm, (xem [2], trang 12).

Dể bài toán có nghĩa ta giả thiết rằng:

$$
A_{1}<R_{1}, A_{1}+A_{2}>R_{1}+R_{2}, S_{1} \geq R_{1}, S_{2} \geq R_{2} .
$$

Từ giả thiết c) suy ra phí tổn trung bình lấy mẫu kiểm tra cũng như tổn thất trung bỉnh của việc chấp nhận hay bác bó một đơn vị sản phẩm là những hàm tuyến tính của chất lượng trung bỉnh $P$ của sản xuất:

$$
\begin{aligned}
& k_{a}(P)=A_{1}+A_{2} P, \\
& k_{r}(P)=R_{1}+R_{2} P, \\
& k_{s}(P)=S_{1}+S_{2} P .
\end{aligned}
$$

$h_{u}(P)$ và $k_{r}(P)$ cắt nhau tại điềm:

$$
P_{0}=\left(R_{1}-A_{1}\right) /\left(A_{2}-R_{2}\right), 0<P_{0}<1 .
$$

Giá trị $P_{0}$ được gọi là mức chất lượng phân biệt. Rõ ràng nếu $P<P_{0}$ thì quyết định chấp nhận sẽ tốt hơn quyết định bác bỏ, còn khi $P>P_{0}$ ta cọ tình trạng ngược lại. 
Nếu $P$ đã biết biết, ta có thể chọn quyết định hoàn toàn đđủng đắn, tồn thất phải chịu khi đó là tồn thát không tránh khỏi:

$$
L_{u}(P)= \begin{cases}k_{a}(P), & \text { néu } P \leq P_{0} \\ k_{r}(P) & \text { nếu } P>P_{0} .\end{cases}
$$

Vì không biết $P$ đối vỡi mỗi lô, ta phài ước lượng nó thông qua việc kiểm tra mẫu. Do các giả thiết a) c), suy ra tồn thất trung bỉnh phải chịu khi sử dụng phương án $(n, c)$ kiĉ̀m tra những lò sản phâm úng với mức chát lượng trung bỉnh $P$ của sản xuất bằng

$$
\begin{gathered}
L(N, n, c, P)=n k_{s}(P)+(N-n)\left\{k_{a}(P) P(P)+k_{r}(P)[1-P(P)]\right\} \\
=n k_{s}(P)+(N-n)\left\{k_{a}(P) P(P)+k_{r}(P) Q(P)\right\},
\end{gathered}
$$

trong đó $P(P)$ và $Q(P)$ làn lượt là xác suát trung bỉnh của việc chấp nlận và bác bỏ.

$$
\begin{gathered}
Q(P)=1-P(P) \\
P(P)=B(n, c P)=\sum_{x=0}^{c} b(n, x, P)=\sum_{x=0}^{c}\left(\begin{array}{l}
n \\
x
\end{array}\right) P^{x}(1-P)^{n-x}
\end{gathered}
$$

với $x$ là thứ phẩm kiểm tra thấy trong mãu.

Dĩ nhién ta luón có

$$
L(N, n, c, P) \geq L_{u}(P), \forall P \in[0,1] .
$$

IIiệu

$$
L_{a}(N, n, c, P)=L(N, n, c, P)-L_{u}(P)
$$

được gọi là tổn thát thặng dư trung bỉnh do sư dụng phương án $(n, c)$.

Như dà biết, khi phân phối $w(P)$ của chát lượng trung bỉnh $P$ của sản xuât là dã biét, phương án kiêm tra cần xác định là phương án Bayes làm cực tiểu tổn thất trung bỉnh toàn cục

$$
L_{w}(N, n, c)=E_{w}\{L(N, n, c, P)\}=\int_{0}^{1} L(N, n, c, P) d w(P) .
$$

Bây giờ già sử

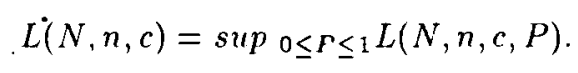

Tir (10) suy ra

$$
L_{w}(N, n c) \leq L(N, n, c) .
$$

Do đó, trong trường hợp không biét phân bớ cuả $P$, nếu chọn $(n, c)$ sao cho $L(N, n, c)$ là nhỏ nhất thì có thể dảm bảo rằng $L_{w}(N, n, c)$ sẽ luôn luôn nhỏ, bất luận $P$ có phân bố như thé nào. 
Dịnh nghĩa Với cỡ lô $N$ cho trước, phương án kiêm tra minimax là phương án $(\hat{n}, \hat{c})$ làm cực tiểu cực đại theo $P$ của hàm tồn thát trung bình $L(N, n, c, P)$ cho trong (6), tức

$$
L(N, \hat{n}, \hat{c})=\inf f_{(n, c)} L(N, n, c)=\inf f_{(n, c)} \sup _{P} L(N, n, c, P) .
$$

Do $L(N, n, c, P)$ là hàm liên tục trên khoảng đóng $[0,1]$ và tập $S$ các phương án có thể chọn là hữu hạn, cụ thể là

$$
S=\{(n, c): 0 \leq n \leq N,-1 \leq c \leq n\}
$$

phương án minimax luôn luôn tồn tại.

\section{Một sổ tính chất và thuật toán xác định phương án minimax.}

Hiên nhiên phương án minimax $(\hat{n}, \hat{c})$ cũng chính là phương án làm cực tiểu cực đại theo $P$ của phiếm hàm sau đây

$$
R(N, n, c, P)=\left[L(N, n, c, P)-L_{u}(P)\right] /\left(A_{1}-A_{2}\right)
$$

$R(N, n, c, P)$ được gọi là hàm tôn thất trung bình đã chuẩn hóa.

Từ (5), (6) và (7) suy ra

$R(N, n, c, P)= \begin{cases}n\left(\delta_{1}+\delta_{2}+P_{0}-P\right)+(N-n)\left(P_{0}-P\right)[1-B(n, c, P)] & \text { nếu } 0 \leq P \leq P_{0} \\ n\left(\delta_{1}+\delta_{2} P\right)+(N-n)\left(P_{0}-P\right) B(n, c, P) & \text { nếu } P_{0} \leq P \leq 1,\end{cases}$

trong đó $\delta_{1}=\left(S_{1}-R_{1}\right) /\left(A_{2}-R_{2}\right) \geq 0$ và $\delta_{2}=\left(S_{2}-R-2\right) /\left(a_{2}-R_{2}\right) \geq 0$, do giả thiết $(2)$.

Để xác định $(\hat{n}, \hat{c})$, có thể thực hiện là̀n lượt hai bước sau đây

- Ứng với mỗi $0 \leq n \leq N$, tìm số chấp nhận minimax $c(n)$.

'- So sánh các phương án $(n, c(n)), n=1,2, \ldots, N$.

Tuy vậy dễ dàng thấy rằng khối lượng tính toán khi đó sẽ rất lớn. Dưới đày sẽ nghiên cứu một số tính chất của phưong án minimax và của hàm tởn thất (13). Các tính chất này sẽ cho phép giảm bớt đáng kể thời gian và không gian tính toán.

Kủ hiệu

$$
R(N, n, c)=\sup _{0 \leq P \leq 1} R(N, n, c, P) .
$$

Bố đề 1. Xét các phương án dặc biệt $(0,0)$ và $(0,-1)$, (không lấy mẫu kiếm tra mà luôn chấp nhận và bác bó một cách tương ứng). Theo nghia minimax, phương án $(0,0)$ tốt hơn phương án $(0,-1)$ nếu $P_{0}>1 / 2$ và tôi hơn trong trường hợp ngược lại.

Chứng minh Với $(0,0)$ và $(0,-1)$ ta có:

$$
\begin{aligned}
& R(N, 0,0, P)= \begin{cases}0 & \text { nếu } P \leq P_{0}, \\
N\left(P-P_{0}\right) & \text { néu } P>P_{0} .\end{cases} \\
& R(N, 0,-1, P)= \begin{cases}N\left(P-P_{0}\right) & \text { nếu } P \leq P_{0} \\
0 & \text { nếu } P>P_{0} .\end{cases} \\
& R(N, 0,0)=N\left(1-P_{0}\right), \quad R(N, 0,-1)=N P_{0} .
\end{aligned}
$$

Từ đây suy ra kết luận của bổ đề. 
Bố để 2 Mọi phương án $(N, c)$ với $0<c \leq N$ không thể là phương án minimax.

Chứng minh. Ta có

$$
\begin{gathered}
R(N, N, c, P)= \begin{cases}N\left(\delta_{1}+\delta_{2} P+P_{0}-P\right) & \text { nếu } P \leq P_{0}, \\
N\left(\delta_{1}+\delta_{2} P\right) & \text { nếu } P>P_{0} .\end{cases} \\
R(N, N, c)=\operatorname{Max}\left\{N\left(\delta_{1}+P_{0}\right), N\left(\delta_{1}+\delta_{2}\right)\right\} .
\end{gathered}
$$

Vì $R(\mathrm{~V}, N, c)$ luôn lớn hơn $R(n, o,-1)=N P_{0}$, ta thu dược két luận nêu trong bồ để.

Bố để 3 Xét các phương án thuộc tập

$$
S^{1}=\{(n, n): n=0,1, \ldots, N-1\} .
$$

Néu $\delta_{1}+\delta_{2}+P_{0}-1 \geq 0$ thì phương án tốt nhất là $(0,0)$. Trường hợp ngược lại, phương án tốt nhất sẽ là $\left(n^{\prime}, n^{\prime}\right)$ với

$$
n^{\prime}=\operatorname{INT}\left\{N\left(1-P_{0}\right) /\left(1-\delta_{2}\right)\right\},
$$

trong dó $\{x\}$ ki hiệu phần nguyèn củi $x$.

Cứng minh. Ta có

$$
R\left(N, n, c, P^{\prime}\right)= \begin{cases}n\left(\delta_{1}+\delta_{2} P+P_{0}-P\right) & \text { nć̉u } P \leq P_{0} \\ n\left(\delta_{1}+\delta_{2} P\right)+(N-n)\left(P-P_{0}\right) & \text { né́u } P>P\end{cases}
$$

Suy ra

$$
\begin{aligned}
& R(, N, n, n)=\max \left\{n\left(\delta_{1}+P-0\right), n\left(\delta_{1}+\lambda_{2} P_{0}\right), n\left(\delta_{1}+\delta_{2}\right)+(N-n)\left(1-P_{0}\right)\right\} \\
= & \max \left\{n\left(\delta_{1}+P-0\right), n\left(\delta_{1}+\delta_{2} P_{0}\right), n\left(\delta_{1}+\delta_{2}+P-0-1\right)+(N-n)\left(1-P_{0}\right)\right\} .(18)
\end{aligned}
$$

- Néu $\delta_{1}+\delta_{2}+P-0-1 \geq 0 R(N, n, n)$ sẽ là hàm tăng của $n$ bất luận với $0<P_{0}<1$ và $\delta_{1}, \delta_{2} \geq 0$. Do đó phương án tớt nhát trong só các phương án thuộc $S^{1}$ phải là $(0,0)$.

- Ngrrợc lại, nću $\delta_{1}+\delta_{2}+P-0-1<0 R(N, n, n)$ sẽ là hàm giảm klì $n \leq n^{\prime}$ và tăng khi $n \geq n^{\prime}$. Do đó plương án tót nhát phài là $\left(n^{\prime}, n^{\prime}\right)$ với $n^{\prime}$ cho trong (16).

Bố đề 4. Nếu chi xét các phurơng án thuợc tập

$$
S^{(2)}=\{(n,-1): n=0,1, \ldots, N-1\} .
$$

thì phương án tớt nhất là $(0,-1)$.

Chứng minh. Ta có

$R(N, n,-1, P)= \begin{cases}n\left(\delta_{1}+\delta_{2} P\right)+N\left(P_{0}-P\right) & \text { nếu } P \leq P_{0}, \\ n\left(\delta_{1}+\delta_{2} P\right) & \text { nếu } P>P_{0} .\end{cases}$

suy ra

$$
R(N, n,-1)=\max \left\{n \delta_{1}+N P_{0}, n\left(\delta_{1}+\delta_{2}\right)\right\} .
$$

Vì $\delta_{1}, \delta_{2} \geq 0, R(N, n,-1)$ luôn luôn là hàm tăng của $n$, do đó phương án tớt nhất thuộc $S^{(2)}$ sẽ là $(0,-1)$. 
Từ các bồ đề 1,3 và 4 ta có kết luận sau đây.

Định lý 1 . Xét các phương án thuộc tập

$$
S^{(3)}=S^{(1)} \cup S^{(2)} \cdot(21)
$$

- Nếu $\delta_{1}+\delta_{2}+P-1 \geq 0$, thì khi đó phương án tốt nhất là $(0,0)$ hay $(0,-1)$ tùy thuộc vào $P_{0}$ lớn hơn hay nhỏ hơ $1 / 2$.

- Nếu $\delta_{1}+\delta_{2}+P-1<0$, phương án tốt nhất là $\left(n^{\prime}, n^{\prime}\right)$ hay $(0,-1)$ tùy theo $\left(1-P_{0}\right)\left(\delta_{1}+P_{0}\right)$ nhỏ hơn hay lớn $P_{0}\left(1-\delta_{1}\right),\left(n^{\prime}\right.$ xác định như trong (16)).

Ký hiệu

$$
\begin{aligned}
& R_{1}(N, n, c)=\text { sup } 0 \leq P \leq P_{0} R(N, n, c, P), \\
& R_{2}(N, n, c)=\text { sup }_{P_{0} \leq P \leq 1} R(N, n, c, P),
\end{aligned}
$$

Bố đê 5 Úng với $N$ và $n$ cố định, $R_{1}(c)=R_{1}(N, n, c)$ là hàm giảm còn $R_{2}(c)=R_{2}(N, n, c)$ là hàm tăng của $c$.

Chứng minh. Bổ để suy ra từ bất đẳng thức sau đay

$$
R(N, n, c, P)= \begin{cases}-(N-n)\left(P_{0}-P\right) b(n, c+1, P) & \text { khi } P \leq P_{0} \\ (N-n)\left(P-P_{0}\right) b(n, c+1, P) & \text { khi } P>P_{0}\end{cases}
$$

Dịnh lý 2. Dặt

$$
n_{1}= \begin{cases}N\left(1-P_{0}\right) /\left(1-\delta_{2}\right) & \text { néu } 0 \leq \delta_{2} \leq P_{0}, \\ n P_{0} / \delta_{2} & \text { nếu } \delta_{2}>P_{0} .\end{cases}
$$

Khi đó phương án với cõ mẫu $n$ thóa $n_{1}<n<N$ sẽ không thể là phương án minimax.

Chứng minh.

a) Già sử $n_{1}=N\left(1-P_{0}\right) /\left(1-\delta_{2}\right)$, khi đó nếu $n>n_{1}$ thì

$$
\begin{aligned}
R_{1}(N, n, n)=n\left(\delta_{1}+P\right) & \geq n\left(\delta_{1}+\delta_{2}+P_{0}-1\right)+N\left(1-P_{0}\right) \\
= & R_{乙}(N, n, n) .
\end{aligned}
$$

Vì $R_{1}(N, n, c)$ là hàm giảm, $R_{2}(N, n, c)$ là hàm tăng của $c$, ta lại có

$$
R_{1}(N, n, c) \geq R_{1}(N, n, n) \geq R_{2}(N, n, n) \geq R_{2}(N, n, c) \geq
$$

với mọi $c$. Suy ra số chấp nhận minimax $c(n)$ ứng với $n$ bằng chính $n$. Theo bồ đê 2 phương án $(n, n)$ với $n>n_{1}$ không thể là phương án minimax.

b) Nếu $n_{1}=N P_{0} / \delta_{2}$, khi đó với $n>n_{1}$ ta có

$$
\begin{gathered}
R_{1}(N, n,-1)=\max \left\{n \delta_{1}+N P_{0}, n\left(\delta_{1}+\delta_{2} P_{0}\right)\right\} \\
\leq n\left(\delta_{1}+\delta_{2}\right)=R_{2}(N, n,-1) .
\end{gathered}
$$

Nhưng theo bồ đề 5 , khi dó ta lại có

$$
R_{1}(N, n, c) \leq R_{1}(N, n,-1) \leq R_{2}(N, n,-1) \leq R_{2}(N, n, c)
$$

với mọi $c$. Suy ra số chấp nhận minimax $c(n)$ ứng với $n$ bằng -1 . Theo bổ đề phương án $(n,-1)$ với $n>N P_{0} / \delta_{2}>0$ không thể là phương án minimax. 
Các kết quả trên đây cho phẹp giảm bớt đáng kể khối lượng công việc tính toán tìm kiếm phương án minimax $(\hat{n}, \hat{c})$. Thay cho tập $S$ bây giờ chî cẩn xít tập

$$
S^{\prime}=\left\{(n, c): n=1,2, \ldots, n_{1} ; 0 \leq c<n\right\} .
$$

Một khi đã tìm được phương án tót nhất thuộc tập $S^{\prime}$ (theo hai bước đã nèu ở đầu mục), đem so sánh với phương án tốt nhất trong số 3 phương án $(0,0),(0,-1)$ và $\left(n^{\prime}, n^{\prime}\right)$ sẽ thu được $(\hat{n}, \hat{c})$.

Vởi các phương án thuộc tập $S^{\prime}$, một lần nữa ta có thể giảm bớt khối lượng tính toán nhờ mệnh đề sau dây.

Dịnh lý 3. V'ới các phương án thuộc tập $S^{\prime}$, hàm tốt nhất (13) có tối da một cực đại địa phương trong mới khoảng $\left(0, P_{0}\right)$ và $\left(P_{0}, 1\right)$.

Chứng minh. Ta có:

$$
\begin{gathered}
B^{\prime}(n, c, P)=-\beta(P, n+1, n-c)=n b(n-1, c, P), \\
B^{\prime \prime}(n, c, P)=\beta(P, c, n-c-1)\{n(P-h) /[h(1-h)]\},
\end{gathered}
$$

trong dó $\beta(x, s, t)$ là hàm mật độ chìa phàn bó Beta với các tham số $s, t$ và $h=c /(n-1)$, (xem [2], trang 192).

Sừ các cong thức trên thu được

$$
\begin{gathered}
R^{\prime}(P)= \begin{cases}n\left(\delta_{2}-1\right)+(N-n)\left\{\left(P_{0}-P\right) n b(n-1, c, P)-[1-b(n, c, P)]\right\} & \text { khi } P \leq P_{0} \\
\left.n \delta_{2}+(N-n)\left\{P_{0}-P\right) n b(n-1, c, P)+B(n, c, P)\right\} & \text { khi } P>P_{0}\end{cases} \\
\left(\begin{array}{c}
R^{\prime \prime}(P)=(N-n) n(n-1 \\
c) P^{c-1} Q^{n-c-2}\left\{(n+1) P^{2}-\left(c+2-P_{0}+n P_{0}\right) P+c P_{0}\right\}
\end{array}\right)
\end{gathered}
$$

Vì $(N-n) P^{2}-\left(c+2-P_{0}+n P_{0}\right) P+c P_{0}$ là tam thức bậc hai của $P$, chỉ tồn tại tối da hai nghiệm $0 \leq x_{1}<P_{0}<x_{2}$. Suy ra $R^{\prime \prime}(P)$ dổi dấu nhiều nhát hai lần trong khoảng $(0,1)$. Hơn nữa, dễ dàng thấy rằng $R^{\prime \prime}(P)<0$ khi $x_{1}<P<\min \left\{x_{2}, 1\right\}$. Do đó $R(P)$ chỉ có cực đại địa phương trong khoảng $\left(0, P_{0}\right)$ khi và chi khi $R^{\prime}\left(X_{1}\right)>0>R^{\prime}\left(P_{0^{-}}\right)$và có cực đại địa phương trong khoàng $\left(P_{0}, 1\right)$ khi và chì khi $R^{\prime}\left(P_{0}+\right)>0>R^{\prime}\left(\min \left\{x_{2}, 1\right\}\right)$.

Đé xác định cực đại địa phương cần giài phương trỉnh $R^{\prime}(P)=0$. Việc này có thể tlực hiện bằng một phương phạp lặp, xuất phát từ nghiệm gần đủng nào đó. Dồng thời, nĉ́u có chương trình tính các cực đại dịa phương này thi việc xác định $R(N, n, c)$ có thể tiến hành một cách dễ dàng:

$$
R(N, n, c)=\max \left\{R_{\ell}(N, n, c), R(N, n, c, 0), R\left(N, n, c, P_{0}\right), R(N, n, c, 1)\right\}
$$

trong dó $R_{\imath}(N, n, c)$ là giá trị lớn hơn trong hai cực trị địa phương.

Dịnh lý 4. Cho cõ̃ mẫu $1 \leq n \leq N-1$, khi đó số chấp nhận minimax $c(n)$ được cho bởi

$$
c(n)= \begin{cases}c_{0}-1 & \text { nếu } R_{1}\left(N, n, c_{0}-1\right) \leq R_{2}\left(N, n, c_{0}\right), \\ c_{0} & \text { néu } R_{1}\left(N, n, c_{0}-1\right)>R_{2}\left(N, n, c_{0}\right),\end{cases}
$$


trong đó co là số nguyên dương nhỏ nhất thỏa mãn

$$
R_{2}(N, n, c)>R_{1}(N, n, c) .
$$

Chứng minh. Vì $R_{1}(N, n, c)$ là hàm giảm, còn $R_{2}(N, n, c)$ là hàm tăng của $c$ (theo bổ đề 5 ), suy ra

$$
\begin{gathered}
\operatorname{Min}_{c}\left\{\operatorname{Max}\left\{R_{1}(N, n, c), R_{2}(N, n, c)\right\}\right\}= \\
\operatorname{Min}\left\{R_{1}\left(N, n, c_{0}-1\right), R_{1}\left(N, n, c_{0}\right)\right\} .
\end{gathered}
$$

Do đó, $c(n)$ được xác định như trong (23).

\section{4. vídu.}

Các kết quả nghiên cứu thu được trong phần 3 cho phép giảm bớt đáng kể không gian và thời gian tỉnh toán xác định phương án minimax. Dưới dây là hai ví dụ minh họa.

Ví dụ 1. Giả sứ $N=200, P_{0}=0,03, \delta_{1}=0,02, \delta_{2}=0,60$.

Vì $P_{0}<\delta_{2}$ nên theo định lý 2 chỉ cần xét các phương án có cỡ mẫu không lớn hơn

$$
n_{1}=N P_{0} / \delta_{2}=2000.0,03 / 0,06=100
$$

IIơn nữa theo định lý 1 , trong số các phương án thuộc tập $S^{(3)}$ phương án tốt nhất là $(0,-1)$.

Sử dụng các kết quả nêu trong định lý 3 và 4 , lạp một chương trỉnh máy tỉnh, dễ dàng tỉm dược một phương án tốt nhất thuộc tập $S^{\prime}$ là $(25,0)$.

Vì $(25,0)$ tốt hơn $(0,-1)$ nên phương án minimax cần xác định là $(25,0)$.

Vi du 2. Cho $N=1500, P_{0}=0,35, \delta_{1}=0,05, \delta_{2}=0$.

Do $\delta_{2}<P_{0}$ nên theo định lý 2 chỉ cần xẹ́t phương án có cỡ mẫu không lớn hơn

$$
n_{1}=\left(1-P_{0}\right) N /\left(1-\delta_{2}\right)=0,65 \times 1500=975 .
$$

Phương án tốt nhất thuộc $S^{(3)}$ là $(975,975)$.

Phương án tốt nhất thuộc $S^{\prime}$ là $(50,17)$.

Vì $(50,17)$ tốt hơn $(975,975)$ nên phương án minimax cần tìm là $(50,17)$.

\section{Tài liêu tham kháo}

1. Colanni, E.V., A sampling system for inspection by attributes. Frontier in Statist. Qual. Contr. 2., ed. by Lenz et al. Physica - Verlage, 1984.

2. Hald, A., Statistical Theory of Sampling Inspection by attributes. Academic Press, London, 1981.

3. Thry regod, P., Towards an algorithm for the minimax regret single sampling strategy. Duplicated report, Institute of Mathematical Statistics, University of Copenhagen, 1969.

4. Uhlmann, W., Kosten - optimal prufplane fur die Gutschlecht - Prufung. Metrica, 13(1968), 206-224. 
5. Van der Vaerden, B.L., Sampling inspection as minimum loss problem. Ann. Math. Statist., 31 (1960), 369-384.

6. Nguyễn Thanh Tùng, Tiếp cận bằng quyết định thống kê bài toán lấy mẩu kiểm tra nghiệm thu, Institute of Math. and Institute of Computer Sci. and Cybern. Preprint series $\mathrm{n}^{\circ} 4 \mathrm{~b}$ (1988).

7. Wetherill, G.B. \& Chiu, W.K., A review of acceptance sampling schemes with emphasis on the economic aspect,Intern. Statist. Review, 43 (1975), 291-310.

8. Feller, W., An Introduction to Probability Theory and its Application, vol. 1, (1968), $3^{\text {th }}$ edn. New-York.

\section{Abstract}

On the problem of design of minimax - regret sampling plans for inspection

The purpose of this paper is to investigatc the problem of designing minimax-regret attributes sampling plans under the following assumptions:

- The production process in control with process average varies at random from lot to lot and othing is known about this variation.

- Two decisions which will be considered regarding the remaider of lot are. acceptance and rejection.

- The per item costs for inspection, acceptance and rejection are all linear functions of the fraction defective in lot.

We study some properties of the optimal sampling plans. These properties are used to devise an improved and efficent algorithm for determination of such plans. Numerical examples are presented to illustrate the potential computational savings of the algorithm. 